\title{
Utilizzo di nuovi test immunologici nella diagnostica dell'infezione da M. tuberculosis
}

\section{Ilaria Sauzullo', Fabio Mengoni', Miriam Lichtner', Raffaella Rossi', Donatella Vincenti², Stefania Carrara ${ }^{2}$, Delia Goletti' ${ }^{2}$,Enrico Girardi ${ }^{2}$, Claudio Maria Mastroianni',Vincenzo Vullo'}

'Dipartimento di Malattie Infettive e Tropicali, Università “La Sapienza” di Roma;

'Laboratorio di collegamento tra ricerca di base e Clinica e II divisione dell'Istituto Nazionale di Malattie Infettive L. Spallanzani di Roma

Key words: M. tuberculosis, TB-Gold, ESAT-6, CFP-I0, IFN-g.

New immune-based assays as an aid for the diagnosis of $M$. tuberculosis infection: role in clinical practice

\section{SUMMARY}

New in vitro test, the Interferon- $\gamma$ assay, has now emerged for diagnosis of latent tuberculosis infection (LTBI). Newer assays based on the immune response to ESAT-6 and CFP-I0 antigens from the RDI of M. tuberculosis may have advantages over TST, in terms of higher specificity, better correlation with exposure to M. tuberculosis and less cross-reactivity due to BCG vaccination and non-TB mycobacterial infection. Two commercially available tests have been approved for the diagnosis of TB infection, the QuantiFERON®TB-Gold and the T Spot.TB. These assays are based on overlapping peptides of ESAT-6 and CFP-10; however, they do not discriminate between A-TB and LTBI. Differently, using multiepitopic peptides from ESAT-6 and CFP-I 0 proteins selected by quantitative implemented HLA peptide-binding motifs analysis, it has bee shown a positive response to RDI selected peptides only in patients with A-TB. Aim of this study is to evaluate the test TB-Gold as a tool to identify TB infection in a population enrolled with suspicious active TB. Agreement between TST and Interferon- $\gamma$ assay $(80 \%)$, rather then sensivity and specifity was reported. Moreover the assay based on RDI selected peptides was used to evaluate whether it would allow a discrimination between active TB and LTBI. Our study suggest, that TB-Gold is useful tool to identify TB infection in a population enrolled with suspicious active TB. Moreover the assay based on RDI selected peptides is a useful tool to discriminate between active TB and LTBI with a positive predictive value of $100 \%$ and a negative predictive value of $76 \%$.

\section{INTRODUZIONE}

La tubercolosi (TB) è l'infezione più diffusa al giorno d'oggi. Si stima che due miliardi di persone - circa un terzo dell'intera popolazione mondiale - siano affette da infezione tubercolare latente (LTBI), senza mostrare sintomi attivi. Nel mondo ogni anno vengono diagnosticati circa otto milioni di nuovi casi di malattia attiva (ATB), che conducono a due milioni di decessi per tubercolosi (6).

La tubercolosi è quindi la più importante causa di morte da singolo agente infettivo, ed è tornata ad essere un problema di sanità pubblica anche nei paesi occidentali. Non vi è dubbio che nel determinismo della attuale situazione sono coinvolti nuovi fattori di rischio che si sono venuti a delineare in tempi recenti come fenomeni inarrestabili e non facilmente controllabili. Uno di questi, di sempre più rilevante portata, è rappresentato dalle forti correnti migratorie da paesi ad alta endemia tubercolare di soggetti che peraltro vivono nella maggioranza dei casi in condizioni socio-sanitarie di emarginazione. Altri fattori sono costituiti dalla diffusione dell'infezione HIV con conseguente alta incidenza di coinfezioni tubercolari, dall'aumento di terapie immunodepressive in pazienti con malattie del sistema immunitario, neoplasie o nei trapiantati $(5,20)$. In ultimo l'emergenza di ceppi micobatterici farmaco-resistenti molto diffusi in alcune aree del mondo causa gravi ripercussioni nella scelta della terapia e nel controllo dell'infezione (7).

La possibilità di rilevare accuratamente e rapidamente l'infezione da $M$. tuberculosis è cruciale per un controllo globale della malattia. La diagnosi ed il trattamento ove indicato dell'infezione tubercolare latente sono considerate priorità assolute per il controllo e l'eliminazione della tubercolosi. In particolare l'identificazione precoce di soggetti infetti, a rischio elevato di progressione clinica e di quelli con tubercolosi polmonare attiva, consente di ridurre le fonti di contagio nella popolazione (14).

La diagnosi della malattia attiva è basata essenzialmente sulla valutazione del rischio di infezione, sul quadro clinico-radiologico, sull'esame diretto dei campioni raccolti, sebbene il gold standard per la diagnosi rimane l'identificazione col- 
turale del M. tuberculosis. Diversamente, per diagnosticare l'infezione tubercolare latente, condizione di quiescenza del micobatterio, non è possibile l'isolamento microbiologico. L'infezione tubercolare latente è infatti, un'infezione sub-clinica caratterizzata dall'assenza di segni clinici, batteriologici, radiologici; la sua diagnosi si basa unicamente sulla dimostrazione di una risposta immunitaria ad antigeni micobatterici (3).

Classicamente l'identificazione delle persone con infezione tubercolare latente è sempre stata affidata al test cutaneo tubercolinico, conosciuto anche come intradermoreazione alla tubercolina secondo Mantoux (T.S.T. tuberculosis skin test). Questo test, invariato nella sua esecuzione da oltre 100 anni, prevede l'iniezione nel derma di un derivato proteico purificato (PPD) di M. tuberculosis che provoca una lesione eritematosa e infiltrante nel punto di inoculo che si manifesta tipicamente dopo 48/72 ore. Dal punto di vista immunologico si ha l'evidenza di una reazione di ipersensibilità tipo IV, cellulo-mediata (11).

Il test cutaneo tubercolinico, pur essendo a tutt'oggi il test di riferimento presenta alcune limitazioni, che in parte ne riducono l'utilità clinica. In primo luogo, è un esame eseguito in vivo, che necessita di una visita di ritorno per l'interpretazione del risultato. Presenta una scarsa standardizzazione sia nell'esecuzione che nella valutazione del test da parte dell'operatore. In secondo luogo è gravato da una bassa specificità dovuta alla cross-reattività antigenica esistente tra PPD, BCG (bacillo di Calmette e Guerin) e micobatteri non tubercolari (NTM), tra i quali anche quelli ambientali. Infine presenta una bassa sensibilità in condizioni di immunodepressione, condizione in cui si assiste ad una progressiva perdita della capacità di montare risposte di ipersensibilità ritardata (12).

Inoltre non permette di distinguere tra infezione latente e malattia attiva e non riveste nessun ruolo nel monitoraggio della terapia. Infatti la cutipositività tubercolinica è espressione di un avvenuto primo attecchimento micobatterico nell'organismo ed ha quindi il significato di memoria dell'evento infettante, indipendentemente dalla presenza o meno di una malattia tubercolare $(14,1)$.

Dal momento che la tubercolosi è in aumento nei paesi sviluppati, che l'infezione latente costituisce un fattore di rischio per la manifestazione della malattia e che il test con il PPD è poco sensibile, negli ultimi anni si è cercato di sviluppare nuovi strumenti per la diagnosi dell'infezione tubercolare latente. È stato così proposto un approccio in vitro basato sulla risposta delle cellule $\mathrm{T}$ anziché sugli effetti cutanei dell'interazione tra cellule presentanti l'antigene e le cellule T.
Questo approccio vede il suo razionale nelle caratteristiche del M. tuberculosis, patogeno intracellulare in grado di evocare in vivo una risposta immunitaria cellulo-mediata (risposta di tipo Th-1) con produzione di interferone gamma $($ IFN- $\gamma)(19)$.

In linea generale un soggetto esposto a $M$. tuberculosis presenta nel sangue periferico dei linfociti T CD4 e CD8 specifici che, se opportunamente stimolati in vitro, amplificano la risposta immune verso il $M$. tuberculosis attraverso la produzione di IFN- $\gamma$ (9). Il dosaggio quantitativo della citochina prodotta, mediante un saggio immuno-enzimatico, permette di rilevare l'avvenuto contatto con il micobatterio tubercolare. L'IFN- $\gamma$, quindi può essere considerato come il marcatore dell'avvenuta attivazione di queste cellule T CD4 e CD8 da parte dell'antigene specifico (15).

Oggi abbiamo a disposizione una serie di nuovi test immunologici in vitro per la diagnosi di infezione tubercolare latente: il QuantiFERON ${ }^{\circledR}$ TBGold (TB-Gold), prodotto in Australia, approvato dal FDA americana nel 2001 e introdotto nelle ultime linee-guida del Center for Disease Control and prevention di Atlanta $(16,2)$ e T Spot.TB prodotto dalla inglese Oxford Immunotec, in attesa di approvazione da parte dell'FDA. La novità di questi nuovi test di laboratorio è legata all'utilizzo come stimolo antigenico di peptidi overlappanti, ossia peptidi che ricoprono tutta la sequenza della proteina considerata, nello specifico delle proteine ESAT-6 e CFP-10, codificate dalla regione genomica RD1, presenti in modo peculiare in M. tuberculosis ed assenti, invece, in tutti i ceppi di M. bovis (BCG) e nella maggior parte dei micobatteri non tubercolari (NTM), esclusi M. kansassii, M. szulgai, M. marinum. Questi due prodotti genici di RD-1, ESAT-6 e CFP10, sono i bersagli dei linfociti Th- 1 che producono IFN- $\gamma$ in soggetti con infezione da M. tuberculosis. L'utilizzo di queste due proteine ha consentito di migliorare sia la sensibilità che la specificità per la diagnosi dell'infezione tubercolare latente $(8,17)$. Inoltre recentemente alcuni studi hanno messo in evidenza l'importanza di selezionare peptidi mediante tecnica computazionale per identificare epitopi promiscui dell'Human Leukocyte Antigen (HLA) di classe II per le proteine di CFP-10 ed ESAT-6. In particolare ogni peptide contiene uno o più epitopi capaci di legare almeno 4 diverse specificità dell'HLA-DR, (ii), 2 dell'HLA-DP (iii) e 2 dell'HLA-DQ con un'abilità di legame dell' $80 \%$ rispetto alla massima capacità di legame di ogni allele appartenente all'HLA di classe II. Questi epitopi così selezionati sono capaci di coprire più del 90\% degli aplotipi di HLA di classe II presente nelle diverse popolazioni umane. La risposta a 
questi peptidi da noi selezionati si associa a TB attiva (21) diversamente dalla risposta ai peptidi overlappanti usati nei test commerciali (10) e permette di monitorare l'efficacia della terapia antitubercolare (4).

Il nostro primo obiettivo è stato quello di determinare l'affidabilità del test TB-Gold nell'ambito di un contesto clinico su pazienti con sospetto di malattia tubercolare. Come obiettivo secondario abbiamo cercato di valutare la possibilità di discriminare l'infezione attiva dalla latente utilizzando i peptidi selezionati di RD1 ESAT 6 e CFP10.

\section{MATERIALI E METODI}

Lo studio è stato condotto su 31 soggetti arruolati con sospetto clinico di malattia tubercolare presso il Dipartimento di Malattie Infettive del Policlinico Umberto I "La Sapienza" di Roma nell'arco di tempo di 6 mesi. Le caratteristiche dei pazienti sono descritte nella tabella 1 . Su tutti i pazienti è stato effettuato il test cutaneo tuberco- linico (TST), il QuantiFERON® TB-Gold e il test immunologico con i peptidi selezionati.

I risultati ottenuti sono stati confrontati con i dati clinici raccolti durante l'anamnesi e con gli esiti degli esami microbiologici.

\section{Test QuantiFERON® TB-Gold (Cellestis Ltd, Australia)}

Il test si svolge in due fasi:

- Nella prima fase si procede all'incubazione di quattro aliquote da $1 \mathrm{ml}$ di sangue intero eparinato rispettivamente con gli antigeni ESAT-6 e CFP-10, il mitogeno di controllo e il controllo negativo.

- Nella seconda fase, dopo l'incubazione di 12-18 h a $37^{\circ} \mathrm{C}$, si preleva il plasma e si quantifica l'interferon gamma mediante saggio ELISA. Una curva con IFN- $\gamma$ ricombinata viene effettuata ad ogni determinazione. I risultati dei campioni sono espressi in Unità Internazionali riferite a questa preparazione standard. Il sog-

Tabella I. Caratteristiche dei pazienti inclusi nello studio

\begin{tabular}{|c|c|c|c|c|c|c|c|}
\hline \multirow[b]{2}{*}{$\mathbf{P z}$} & \multicolumn{3}{|c|}{ Caratteristiche } & \multicolumn{4}{|c|}{ Valutazione per singolo paziente } \\
\hline & Età & Sesso & TST & BCG vaccinati & Immigrati* & HIV & Recente Esposizione \\
\hline I & 28 & $M$ & - & & + & & \\
\hline 2 & 30 & $\mathrm{~F}$ & + & + & + & & \\
\hline 3 & 5 & $M$ & + & + & & & \\
\hline 4 & 38 & $\mathrm{~F}$ & + & + & + & & \\
\hline 5 & 7 & $M$ & + & + & + & & + \\
\hline 6 & 6 & $M$ & + & + & & & + \\
\hline 7 & 46 & $M$ & - & & & & \\
\hline 8 & 55 & $\mathrm{~F}$ & - & & & & \\
\hline 9 & 32 & $M$ & + & & & & \\
\hline 10 & 60 & $M$ & + & & & & \\
\hline 11 & 41 & $\mathrm{~F}$ & + & & + & + & \\
\hline 12 & 56 & $M$ & + & & & & \\
\hline 13 & 62 & $M$ & + & & + & & \\
\hline 14 & 44 & $\mathrm{~F}$ & + & & + & + & \\
\hline 15 & 29 & $\mathrm{~F}$ & + & & + & & \\
\hline 16 & 43 & $\mathrm{~F}$ & - & & & & \\
\hline 17 & 4 & $\mathrm{~F}$ & + & & + & & \\
\hline 18 & 7 & $\mathrm{~F}$ & + & & & & + \\
\hline 19 & 2 & $M$ & + & & + & & + \\
\hline 20 & 58 & $M$ & + & & & & + \\
\hline 21 & 1 & $M$ & + & & & & + \\
\hline 22 & 49 & $M$ & + & & & & + \\
\hline 23 & 55 & $M$ & + & & + & & \\
\hline 24 & 36 & $M$ & + & & & & \\
\hline 25 & 86 & $\mathrm{~F}$ & + & & & & \\
\hline 26 & 45 & $M$ & + & & & & \\
\hline 27 & 10 & $M$ & + & & & & + \\
\hline 28 & 69 & $\mathrm{~F}$ & + & & & & \\
\hline 29 & 73 & $M$ & + & & & & + \\
\hline 30 & 52 & $M$ & + & & & & \\
\hline 31 & 6 & $\mathrm{~F}$ & + & & + & & \\
\hline
\end{tabular}


getto è ritenuto positivo per l'infezione da $M$. tuberculosis se la produzione di IFN- $\gamma$ verso gli antigene ESAT-6 o CFP-10 supera il cut-off stabilito di 0.35 Unità Internazionali (UI).

\section{Test immuno-enzimatico con peptidi selezionati di RD1}

Il test viene eseguito con le stesse modalità del Quantiferon-TB Gold ad eccezione degli stimoli utilizzati, costituiti da un pool di peptidi multiepitopici di ESAT-6 e CFP-10 (21). Il cut-off per TB attiva è di $0.6 \mathrm{UI} / \mathrm{ml}$ ed è stato stabilito utilizzando la curva ROC in base a un lavoro precedentemente descritto (10).

\section{RISULTATI}

Nello studio sono stati arruolati 31 soggetti con sospetto di tubercolosi. I risultati sono illustrati sinteticamente in figura I.

Sulla base del quadro clinico-strumentale e della successiva conferma di coltura microbiologica solo in 15 soggetti è stata confermata la diagnosi di malattia tubercolare attiva, 13 tubercolosi polmonari, 1 tubercolosi disseminata e 1 spondilodiscite tubercolare. In tale gruppo il TST è risultato positivo in tutti i pazienti (100\%), mentre il TBGold è risultato positivo in 14/15 (93\%). Il test con peptidi selezionati ha dato esito positivo in 11/15 (73\%). L'indice di concordanza tra TST e TB-Gold è pari al $93 \%$. La produzione di INF- $\gamma$ in risposta agli stimoli antigenici, ESAT-6 e CFP-10 nei 14 soggetti TB-Gold positivi, espressa come media $\pm \mathrm{DS} \mathrm{UI} / \mathrm{ml}$, è rispettivamente di $2.6 \pm 0.1$ e $1.9 \pm 0.3$.

Nel gruppo di pazienti $(n=16)$ in cui il sospetto di tubercolosi polmonare non è stato confermato, 7 $(43 \%)$ sono risultati TST+/TB-Gold+/Peptidi-, indicando un quadro di infezione tubercolare latente. Cinque soggetti (31\%) invece erano TST+/TB-Gold-/Peptidi- e nell'anamnesi era riportata una pregressa vaccinazione con BCG. Infine 4 pazienti $(25 \%)$ erano negativi ai tre test (TST-/TB-Gold-/Peptidi-).

\section{DISCUSSIONE}

Nei 15 soggetti con diagnosi di tubercolosi attiva i nostri dati hanno evidenziato una alta affidabilità del test TB-Gold (14/15). Il soggetto che in fase di malattia tubercolare attiva ha presentato un risultato TB-Gold negativo ha evidenziato un quadro clinico di tubercolosi extra-polmonare (spondilodiscite) molto severo a cui potrebbe essere associato uno scarso rilascio antigene-specifico di INF- $\gamma$ da parte delle cellule immunocompetenti. Nei casi di tubercolosi extra-polmonare è descritta la presenza di falsi negativi in letteratura $(13,22)$, sebbene manchino analisi approfondite per comprendere i meccanismi immunologici responsabili.

La buona interpretazione dei risultati è confermata ulteriormente dalla discriminazione dei risultati positivi rispetto al cut-off. Questo dato è evidenziato dalle risposte quantitative agli stimoli ESAT-6 e CFP-10, in termini di IFN- $\gamma$ prodotto, che risultano essere rispettivamente 7 e 5 volte superiori al cut-off del test di $0.35 \mathrm{UI} / \mathrm{ml}$.

I risultati ottenuti, utilizzando come stimolo antigenico il pool di peptidi selezionati di RD1, mostrano una produzione di IFN- $\gamma$ maggiore nei pazienti con diagnosi di malattia tubercolare attiva rispetto ai soggetti con una probabile infezione tubercolare latente $(4,10,21)$.

In tal modo abbiamo identificato uno stato di malattia tubercolare attiva in 11/15 (73\%) dei soggetti in studio, con una sensibilità e specificità pari a $73 \%$ e $100 \%$ rispettivamente e con un valore predittivo positivo (VPP) e un valore predittivo negativo (VPN) del 100\% e 76\% rispettivamente (tabella 2).

L'assenza di risposta al pool di peptidi selezionati è stata riscontrata solo in $4 / 15$ dei pazienti con TB attiva, tale condizione è riconducibile ad uno stato di " $M$. tuberculosis non responsivo-specifico" che può presentarsi nella fase di massima replicazione del patogeno (18).

Per quanto riguarda i 16 soggetti con sospetto di infezione tubercolare, il risultato dei test ha confermato in 7/16 dei soggetti una condizione clinicamente compatibile con una diagnosi di infezione tubercolare latente (tabella 2).

La discordanza tra TST vs TB-Gold in 5/16 dei soggetti analizzati è correlata ad una precedente vaccinazione con $B C G$ evidenziata nell'anamnesi clinica. Il Quantiferon-TB GOLD ha confermato la capacità di discriminare i falsi-positivi al TST dovuti alla vaccinazione con BCG.

I risultati negativi rilevati in $4 / 16$ dei soggetti sono stati confermati successivamente da diagnosi di patologie non correlate all'infezione tubercolare. La sensibilità e specificità del TB-Gold rispetto al TST calcolata sui 31 soggetti in studio è pari al $77 \%$ e $100 \%$ rispettivamente. Il valore della sen-

Tabella 2. Confronto di differenti test per la diagnosi di TB

\begin{tabular}{lccc}
\hline & & \multicolumn{2}{c}{ N. di pazienti positivi/ totale pazienti (\%) } \\
\cline { 3 - 4 } Peptidi & Test & PZ con TB attiva & PZ con sospetto di TB latente \\
\hline Overlappanti RDI & Quantiferon-TB-Gold & I4/I5 (93) & $7 / 16(43)$ \\
\hline Selezionati RDI & Test immunologico & II/I5 (73) & $0 / 16$ \\
\hline
\end{tabular}


sibilità risulta sottostimato per la presenza nella casistica di 5 soggetti vaccinati con BCG la cui assenza si tradurrebbe in una stima della sensibilità del 95\%.

In conclusione, considerando il limite dell'esiguo numero di soggetti analizzati, i dati estrapolati hanno dimostrato l'ottima affidabilità del test immunologico TB-Gold nella diagnostica dell'infezione da Mycobacterium tuberculosis sia nella forma attiva che nella forma latente.

Inoltre, dato di estrema importanza, l'utilizzo del pool di peptidi selezionati di RD1 ha portato ad identificare la forma di TB-attiva con una buona performace ed un VPP del 100\%.

La rapidità e semplicità di esecuzione dei test immunologici analizzati, li rendono ottimi candidati per essere introdotti nella diagnostica di routine dell'infezione tubercolare.

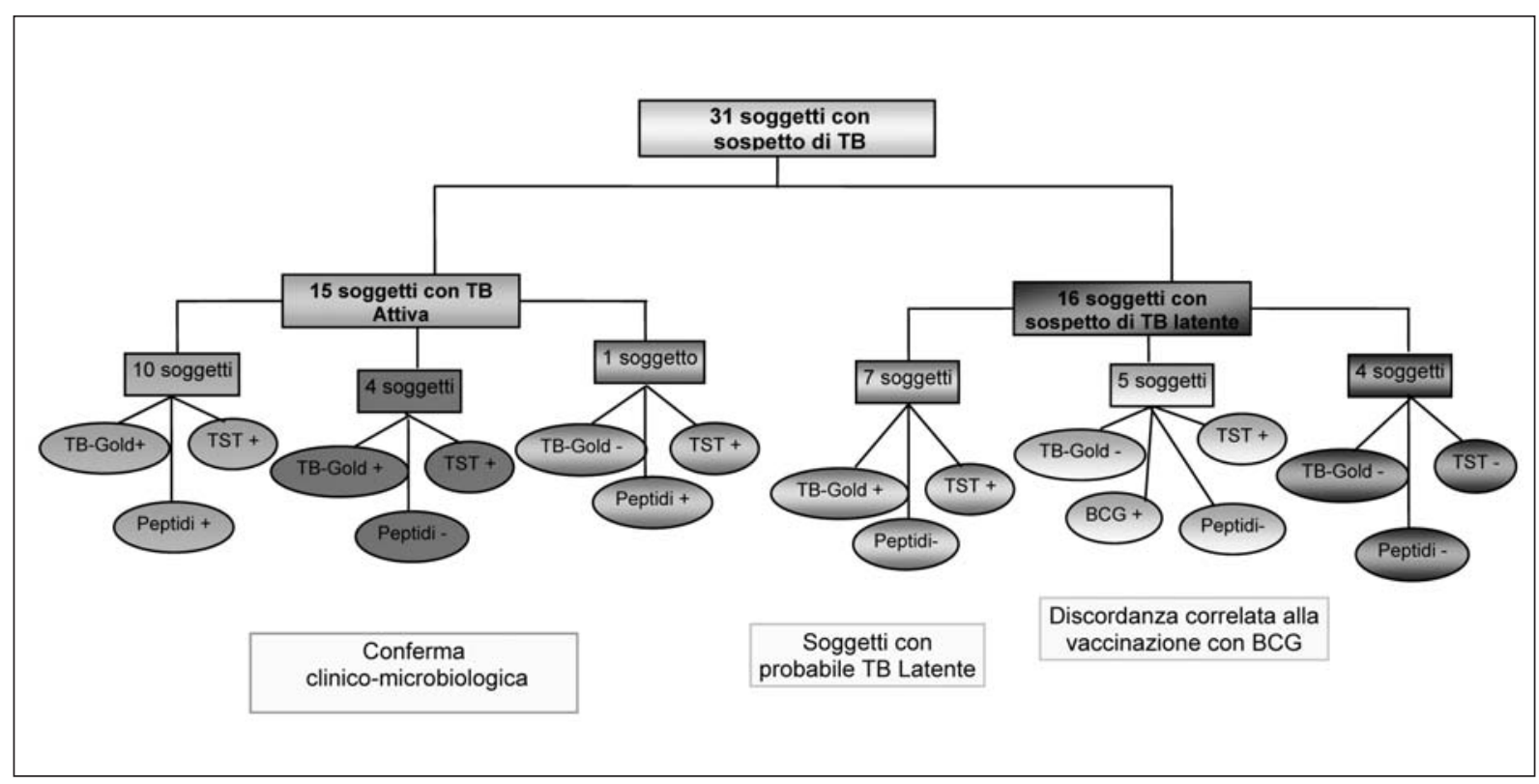

Figura I. I risultati del test QuantiFERONTB-Gold, delle ricerche microbiologiche e cliniche dei 3 I pazienti arruolati con sospetto di TB sono mostrati in questo diagramma. I pazienti con risposta al QuantiFERON TB-Gold $>0.35 \mathrm{Ul} / \mathrm{ml}$ (cut off) sono considerati positivi

\section{BIBLIOGRAFIA}

1. Andersen P, Munk ME, Pollock JM, Doherty TM. Specific immune-based diagnosis of tuberculosis. Lancet 2000; 356: 1099-104.

2. Barclay L, Lie D. CDC Issues Guidelines on use of QuantiFERON TB Gold test. MMWR Morb Mortal Wkly Rep 2005; 54 (RR-15): 49-55.

3. Barnes PF. Diagnosing latent tuberculosis infection: turning glitter to gold. AM J Respir Crit Care Med 2004; 170: 5-6.

4. Carrara S, Vincenti D, Petrosillo N, Amicosante M, Girardi E, Goletti D. A T cell-based assay for monitorino efficacy of antituberculosis therapy. Clin Infect Dis 2004; 38: 754-6.

5. Corbett EL, et al. The growing burden of tuberculosis: global trends and interaction with the HIV epidemic. Arch Intern Med 2003; 163 (9): 1009-21.

6. Dye C, Scheele S, Dolin P, Pathania V, Raviglione MC. Consensus statement. Global Burden of tuberculosis: estimated incidence, prevalence, and mortality By country. Who global surveillance and monitoring project. JAMA 1999; 282: 677-86.

7. Dye C, Espinal MA, Watt CJ, Mbiaga C, Williams BG. Worldwide incidence of multidrug-resistant tuberculosis. J Infect Dis 2002; 185 (8): 1197-202.

8. Ferrari G, Losi M, Meacci M, Mecugni B, Piro R, Richeldi L. Routine hospital use of a new commercial whole blood interferon-g assay for the diagnosis of tuberculosis infection. Am J Respir Crit Care Med 2005; 172: 631-5.

9. Flynn JL, Chan J. Immunology of tuberculosis. Annu Rev Immunol 2001; 19: 93-129.

10. Goletti D, Vincenti D, Carrara S, et al. Selected RD1 peptides for active tuberculosis diagnosis: comparison of a gamma interferon whole-blood enzyme-linked immunosorbent assay and an enzyme-linked immunospot assay.

11. Huebner RE, Schein MF, Bass JB. The tuberculin skin test. Clin Infect Dis 1993; 17: 968-75.

12. Lalvani A, Richeldi L, Kunst H. Interferon gamma assay for tuberculosis. Lancet Infect Dis 2005; 5 (6): 322-4.

13. Lin Y, Zhang M, Hofman FM, Gong J, Barnes PF. Absence of a prominent Th2 cytokine response in human tuberculosis. Infect Immun 1996; 64: 1351-6.

14. Jasmer RM, Nahid P, Hopewell PC. Latent tuberculosis infection. New Eng J Med 2002; 347: 1860-6.

15. Madhukar P, Lee W, Colford JM. Interferon-gamma assays in the immunodiagnosis of tuberculosis: a systematic review. Lancet Infect Dis 2004; 4: 761-76.

16. Mazurek GH, Villarino ME. Guidelines for using the QuantiFERON-TB test for diagnosing latent $\mathrm{M}$. tuberculosis infection. Center for Disease Control and Prevention. MMWR Recomm Rep 2003; 52 (RR-2): 15-18. 
17. Mori T, Sakatani M, Yamagishi F, et al. Specific detection of tuberculosis infection:an inteferongamma-based assay using new antigens. Am J Respir Critic Care Med 2004; 170: 59-64.

18. Nicol MP, Sola C, February B, Rastogi N, Steyn L, Wilkinson RJ. Distribution of strain families of Mycobacterium tuberculosis causing pulmonary and extrapulmonary disease in hospitalized children in Cape Town, South Africa. J Clin Microbiol 2005; 43 (11): 5779-81.

19. Palomino JC. Nonconventional and new methods in the diagnosis of tuberculosis: feasibility and applicability in the field. Eur Respire J 2005; 26: 339-50.

20. Stewart GR, Robertoson BD, Young DB. Tuberculosis: a problem with a persistence. Nat Rew Microbiol 2003; 1: 97-105.

21. Vincenti D, Petrosillo N, Carrara S, Girardi E, Goletti D. Identification of Esat-6 epitopes for the immunodiagnosis of active Tuberculosis. Mol Med 2003; 9: 105-11.

22. Zhang M, Lin Y, Iyer DV, Gong J, Abrams JS, Barnes $\mathrm{PF}$. T cell cytokine responses in human infection with Mycobacterium tuberculosis. Infect Immun 1995; 63: 3231-4.

\section{Sauzullo Ilaria}

Dipartimento di Malattie Infettive e Tropicali, Università "La Sapienza" di Roma, Policlinico Umberto I, 00161 Roma. Tel. 06 49970881; Fax 0649972625 E-mail: ilariasauzullo@libero.it 